\title{
Ant Algorithm for AP-N Aimed at Optimization of Complex Systems
}

\author{
Jerzy Mikulik ${ }^{\star}$, Mirosław Zajdel ${ }^{\star}$
}

\begin{abstract}
Assignment Problem (AP), which is well known combinatorial problem, has been studied extensively in the course of many operational and technical researches. It has been shown to be NP-hard for three or more dimensions and a few non-deterministic methods have been proposed to solve it. This paper pays attention on new heuristic search method for the n-dimensional assignment problem, based on swarm intelligence and comparing results with those obtained by other scientists. It indicates possible direction of solutions of problems and presents a way of behaviour using ant algorithm for multidimensional optimization complex systems. Results of researches in the form of computational simulations outcomes are presented.
\end{abstract}

Keywords: assignment problem, ant algorithm, optimization.

Mathematics Subject Classification: 90C27 - combinatorial optimization, 90C59 - approximation methods and heuristics.

Revised: 05 April 2010.

\section{MULTI-CRITERIA OPTIMIZATION}

\section{1. $\quad N$-DIMENSIONAL ASSIGNMENT PROBLEM}

The problem of assignment belongs to fundamental issues of combinatorial optimization and it is often considered in the spectrum of operational researches or production engineering and management. Varied forms of assignment problem have been adapted to many issues, in which the key aspect is economic factor (Owsinski and Straszak, 2002). Starting with simple staff management, operated by assignment problem with linear quality factor, through the transport of consumer goods and precious stones processing, ending with multi-criteria technical problems connected with costs of specific number of factors correlation (Gorecki, 2006).

In its most simple and commonplace form, assignment problem is a task described in two-dimensional space with linear factor of quality. This means that linear assignment problem can be reduced to searching for maximum association in dichotomic

\footnotetext{
* Department of Management and Systems Engineering, Faculty of Management, AGH University of Science and Technology, jmikulik@zarz.agh.edu.pl; mzajdel@agh.edu.pl
} 
graph. In practice it can usually be considered as an analysis of two sets with power of $\mathrm{n}$ (there are also solutions for sets with different power (Filipowicz, 1998)), for which minimum of cost function is to be found. Algorithms that are able to solve problems defined in this way are immunological techniques, which are pretty good for problems of small and average size. However the most popular and widely applied method is the Hungarian algorithm, which in general is the best method of complexity $O\left(n^{3}\right)$ (Kuhn, 1955).

However there is no such universal method for multidimensional form of assignment problem, which is a little inconvenient, but on the other hand it has an ideal setting in which one can demonstrate skills and is an area for some improvements and analysis based on non-deterministic methods, heuristics and biologically inspired techniques.

Spreading assignment problem with linear quality factor to any finished quantity of dimensions, we have to describe it, basing on $m$ separated sets $X_{1}, X_{2}, \ldots, X_{m}$, among which one usually represents single parameter with separately specified $m-1$ dimensional matrix of costs for all possible combinations between considered parameter and all other parameters.

Then the cost function looks as follows:

$$
f=\sum_{i_{1} \in X_{1}} \sum_{i_{2} \in X_{2}} \ldots \sum_{i_{m} \in X_{m}} c_{i_{1}, i_{2}, \ldots, i_{m}} x_{i_{1}, i_{2}, \ldots, i_{m}}
$$

where:

$c_{i_{1}, i_{2}, \ldots, i_{m}}$ is a cost table of real values,

$x_{i_{1}, i_{2}, \ldots, i_{m}}$ is a desirable table of assignment.

Successive, the following restrictions have to be applied. The first one concerns to normalization in all the $m$ dimensions and so for all the $n$ values in each dimension. This restriction secures the computation for exactly one appearance in one association among all associations.

$$
\forall k \in\{1,2, \ldots, m\}: \sum_{i_{1} \in X_{1}} \sum_{i_{2} \in X_{2}} \ldots \sum_{i_{k-1} \in X_{k-1}} \sum_{i_{k+1} \in X_{k+1}} \ldots \sum_{i_{m} \in X_{m}} x_{i_{1}, i_{2}, \ldots, i_{m}}=1
$$

The second restriction applies to Cartesian product of all the dimensions and is a natural effect of associations definition based on $m$ sets representing dimensions.

$$
\forall\left\{i_{1}, i_{2}, \ldots, i_{m}\right\} \in X_{1} \times X_{2} \times \ldots \times X_{m}: x_{i_{1}, i_{2}, \ldots, i_{m}} \in\{0,1\}
$$

As it has already been mentioned, there are permitted assignment problems with different quantities of variables in sets. Before applying this model to them, such an 
assignment problem has to be reduced to AP with equal number of variables using one of available methods (Filipowicz, 1998).

$$
\left|X_{1}\right|=\left|X_{2}\right|=\ldots=\left|X_{m}\right|=n
$$

Finally the last one restriction forbids placing one variable in more than one dimension and is a suplement for the first restriction.

$$
X_{1} \cap X_{2} \cap \ldots \cap X_{m}=\emptyset
$$

As a result of such definition of the problem, searching for solution consists of searching for maximum association in m-chotomic graph. It is easy to notice that the number of all solutions for $m$ sets of power $n$ is $n !^{m}$, because for each set we are able to point at $n$ ! permutations of its elements. It has already been shown, that even for $m=3$ the problem is NP-hard (Karp, 1972).

Also, assuming that $P \neq N P$, a deterministic algorithm for N-dimensional assignment problem, which could be able to solve it in polynomial time, does not exist. Then, we should rather try to find good algorithms for multidimensional assignment problem among non-deterministic techniques.

\subsection{EXISTING SOLUTIONS}

A few algorithms for multidimensional assignment problem has already been shown. They are mainly focusing on the 3-dimensional form of the problem. Probably because of huge computational complexity of N-dimensional form $(N>3)$ and because of many more real issues for 2- or 3-dimensional form, which is more natural for human. Examples of such issues can be various forms of processing with machine cutting, for which we can solve the AP3 problem and minimize the amount of scrap material.

One can find some proposals of algorithmic solvers for Three-dimensional Assignment Problem (AP3) in many papers. They were presented among others in Balas and Saltzman (1991), Crama and Spieksma (1992), Burkard et al. (1996), Aiex et al. (2005) and Huang and Lim (2006), in which hybrid Local Search Genetic Algorithm (LSGA) has appeared as the best among the ones published so far.

Multidimensional assignment problem for the quantity of dimensions equal 4 or more is rather omitted in literature, despite the fact that the number of its possible applications is still growing. Especially some complex mechanisms, like multi-sensor systems for multiple objects monitoring take advantage of $\mathrm{N}$-dimensional Assignment Problem (AP-N) (Escamilla-Ambrosio and Lieven, 2004). Surely N-dimensional AP can also be an attractive tool for companies, which are able to invest some finance for gathering statistical data, in order to gain optimization of production process, complex networks or some distributed systems management.

However it is worth mentioning, that the number of factors to optimize (number of dimensions for AP) has to be chosen as a result of reasonable choice between expense for researches and the degree of participation of each factor in the cost function. 


\section{IMPROVED ANT ALGORITHM FOR AP-N}

\subsection{COMPUTATIONAL MODEL}

The proposed form of solution is based on reduction m-chotomic graph to the model of kind of network presented in Figure 1, which was created by adding the source node ("Ant hill") connected by the edge of 0 weight with all the $n$ nodes of $X_{1}$ set, and also adding the outlet node ("Food") connected by the edge of 0 weight with all the $n$ nodes of $X_{m}$ set.

The intention of ant algorithms working is made on the basis of communication between single insects in a swarm. The swarm is going out of "Ant hill" to find food.

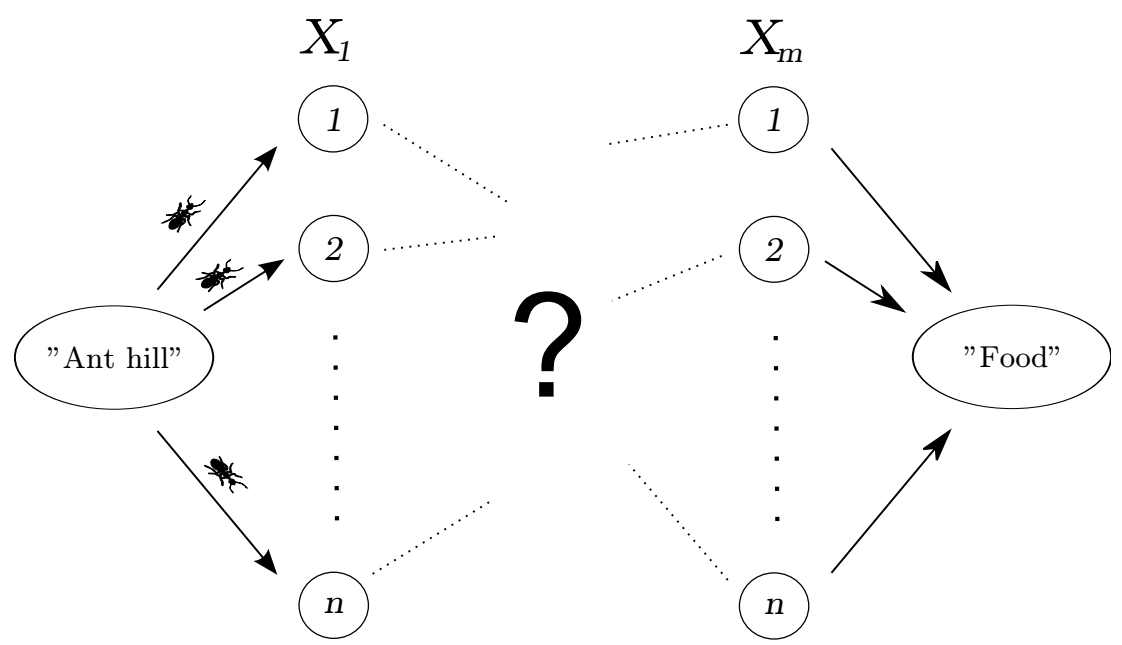

Fig. 1. M-dimensional ant heuristic model for multi-criteria network optimization (Source: Authors' study)

First, all the insects are choosing their paths randomly. When one finds "Food", it comes home marking the path with pheromone, so that all the other insects could smell the pheromone and recognize the profitable path. The pheromone concentration can increase or decrease, depending on the number of insects, which choose that path. After some time ants choose only the paths, which are most profitable from the point of easy food gaining.

Similarly for an assignment problem, after some time, those paths indicate correlations between factors, which are best for the optimization based on function of cost. Taking into consideration restrictions of the model, each element from each set from $X_{1}$ to $X_{m}$ can occur on the single ant path only once. Algorithm uses $n$ ants so after each iteration we get a set of $n$ paths composed of $m$ nodes (omitting the source and the outlet), which set fulfills all the restrictions of assignment problem and for which cost function value is being optimized.

When it comes to changes and improvements made with relation to basic version of algorithm (Zajdel and Filipowicz, 2008), they are mainly:

- Global analysis of pheromone values (Merkle and Middendorf, 2005) - that ability has no reflection in the biological world of insects, but somehow improves the results and significantly shortens the relaxation time of algorithm. 
- Dynamic adaptation of search speed - changes of parameters values connected with effects of intensification and evaporation of pheromone are dynamically adjusted to changes of solutions (cost function values) found in each iteration (the greater changes of cost function towards better solutions, the greater changes of pheromone factors).

- Killing ants after a few steps, which seem to end with solution of rather poor cost function value - behaviour, which is to correspond with situations, when an ant comes up against predator or opponent, loses its way or is exploring little promising areas (thus we save computational power and abilities, eliminating unnecessary searches).

\subsection{SIMULATIONS AND RESULTS}

Computational tests were based on two standard sets of data with various profile of complexity. Those sets were published by teams Balas-Saltzman Balas and Saltzman (1991) and Crama-Spieksma Crama and Spieksma (1992), which have studied assignment problem at the very first years of its development.

Apart from the improved ant algorithm, comparative sheet encompasses also the best techniques worked out and published so far. Since the most detailed and complete were the results in publications concerning meta-heuristic Greedy Randomized Adaptive Search Procedure (GRASP) (Aiex et al., 2005) and hybrid genetic algorithm Local Search Genetic Algorithm (LSGA) (Huang and Lim, 2006), we have chosen those to compare with the ant algorithm (Fig. 2).

Balas-Saltzman dataset

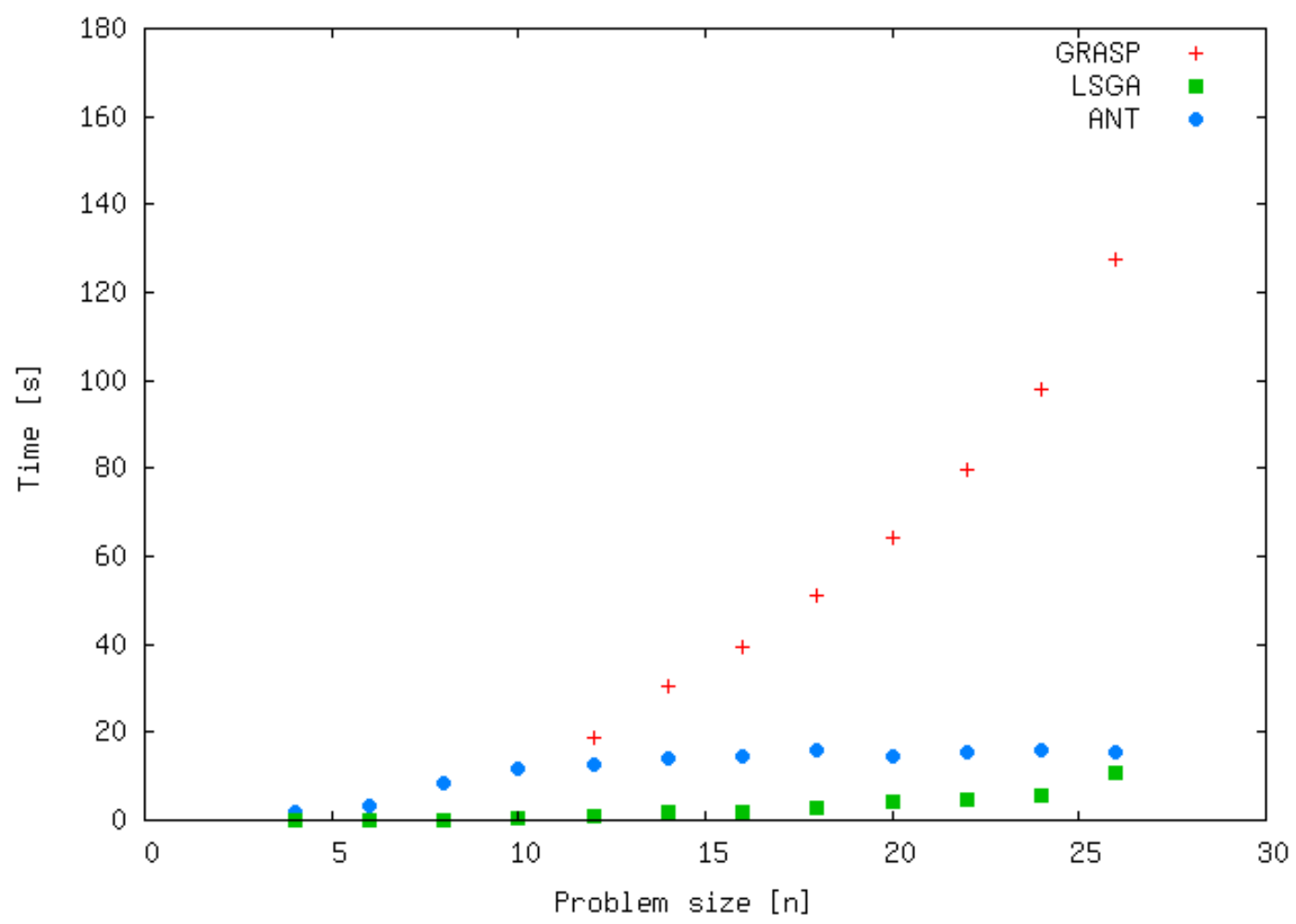

Fig. 2. Comparison of ANT, GRASP and LSGA for small/average AP3 problem size (Source: Authors'study) 
The very first part of computational simulations was based on 60 tests drawn up by team Balas-Saltzman. Balas-Saltzman tests set consists of randomly generated five instances for each problem size of $n=4,6,8,10,12,14,16,18,20,22,24$ and 26. Ant algorithm seems to be far more better than GRASP meta-heuristic. LSGA genetic modifications is still the best, but leads only insignificantly, which we can see in Figure 2. To sum up, small problems do not let the ant algorithm show its strength. Genetic search is slightly faster, because it includes less procedures of preparing the spectrum of search and so in each iteration.

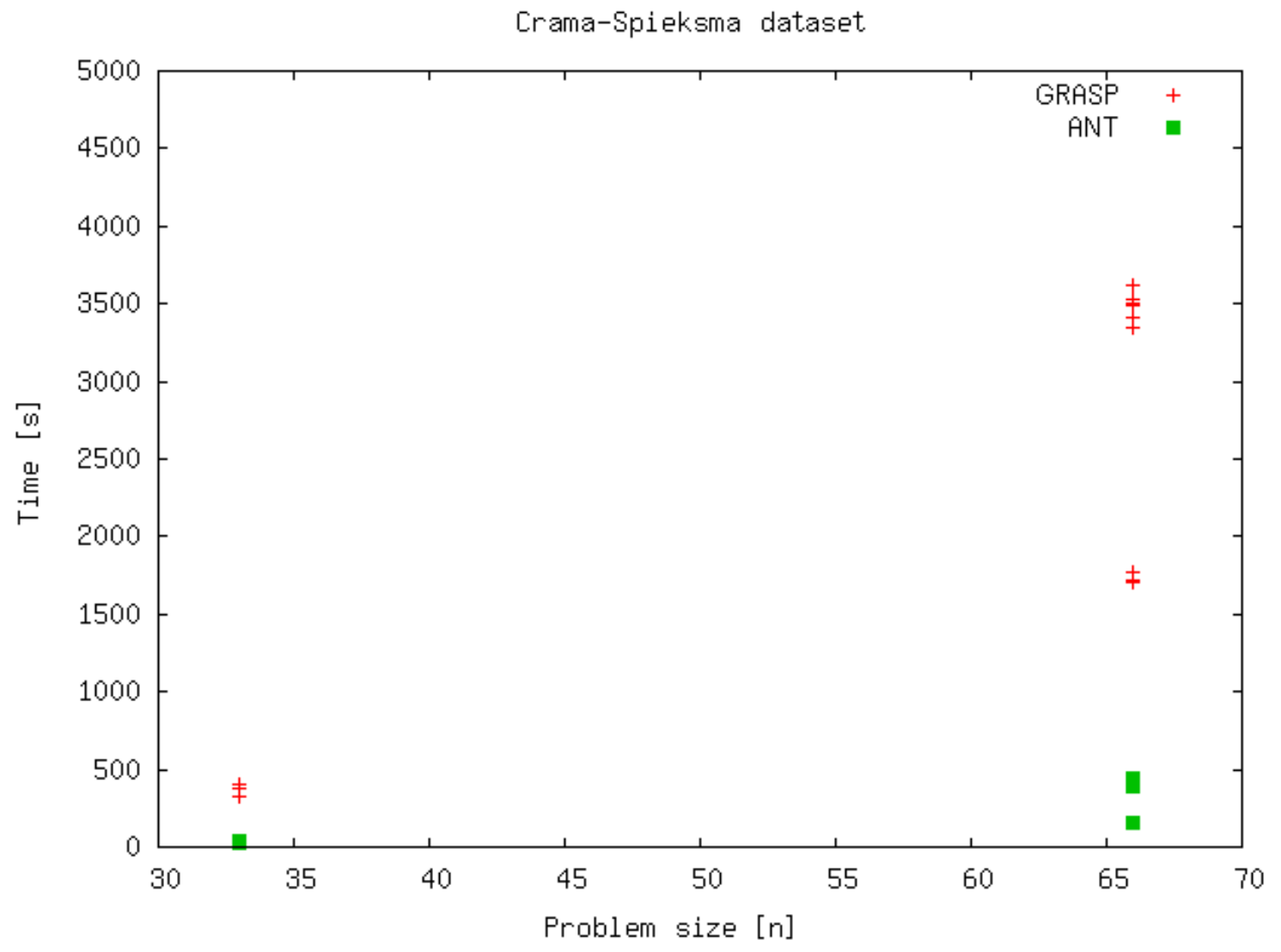

Fig. 3. Comparison of ANT and GRASP for big AP3 problem size

(Source: Authors'study)

For bigger size problems, the situation is spreading. Ant algorithm confirms its high efficiency and good convergence, which is a result of acceleration of search machinery and improving factors, which are able to show their value only after a quantity of first iterations. Test set for problems of big size was taken from team CramaSpieksma. That set consists of 18 tests generated for $n=33$ and 66 . Huang and Lim (2006) did not publish results of LSGA for AP3 of such big size, but profiles of LSGA and ant algorithms from the Figure 3 let us to suspect, that ant algorithm could turn out to be a better one.

Both standard data sets for small and average or big size of AP3 problem are available on site http://www.research.att.com/ ${ }^{\sim m g c r / d a t a / 3 i n d e x . t a r . g z . ~}$ 


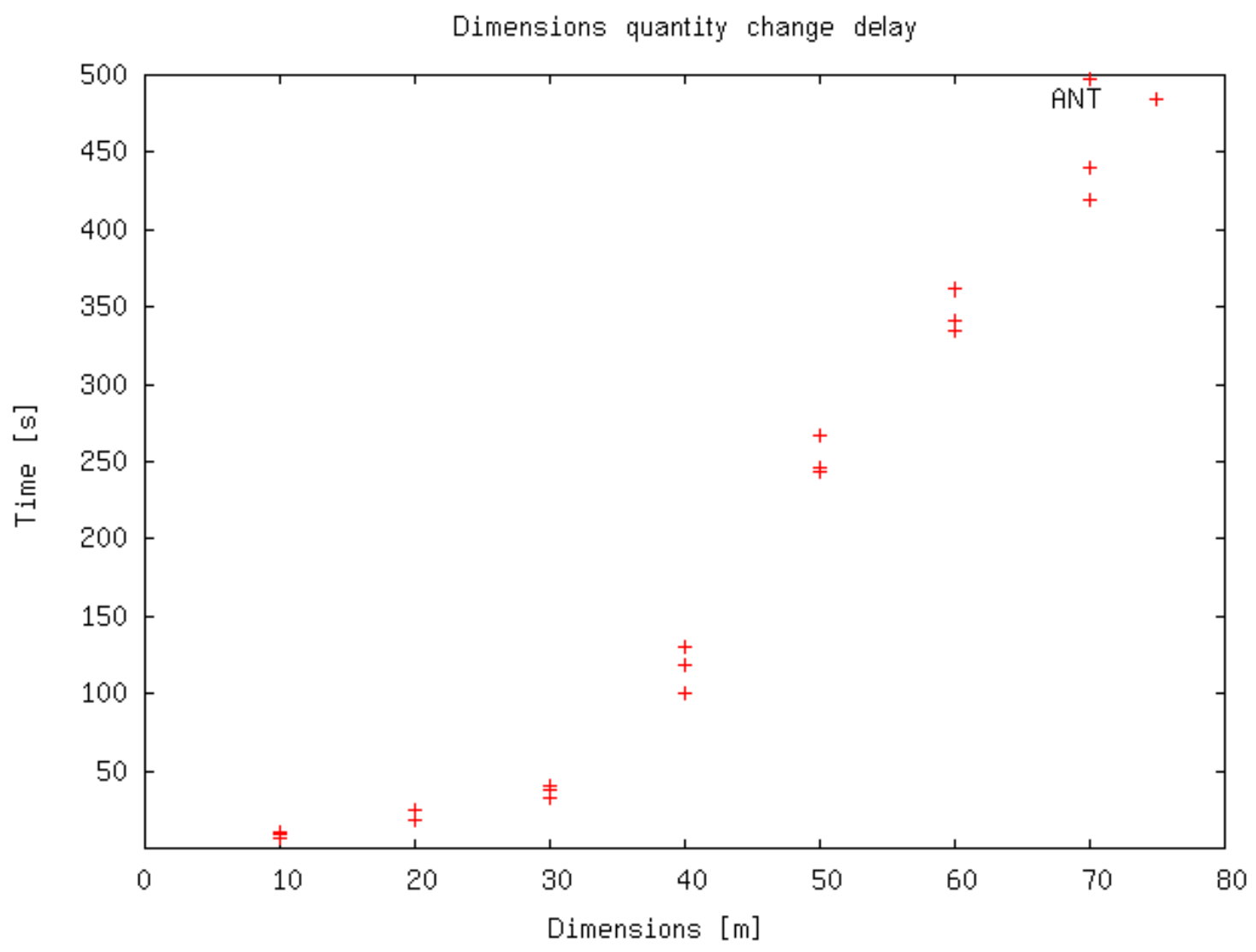

Fig. 4. Asymptotic convergence of $A N T$ in $A P-N$

(Source: Authors' study)

Unfortunately, there are no published results of any algorithm for dimensions number $m>3$, so results for more than three dimensions are only relative for our ant algorithm and point just at its asymptotic convergence, which turned out to be satisfactory. Average results of tests based on randomly generated problems for different number of dimensions are presented in the Figure 4.

\section{SUMMARY}

Summing up, the presented, improved ant algorithm for problems of small and average size is situated between methods of average and good efficiency among techniques published so far. However, for problems of big size $(n \geqslant 30)$ it occurs to have a really good asymptotic convergence. This fact predestines it to be a pretty efficient and useful tool for assignment problems located in a lot of dimensions. Thus, there is a great opportunity to solve most complex combinatorial optimization problems, in which function of cost is dependent on many criteria.

Referring to that case, expectations pointed at designers of telematic networks are going to be more and more complicated and are connected with many factors. At present, when we are still getting new services and solutions, when networks are growing incredibly fast and expectations of clients are rising, designers and analysts 
should still adapt traffic flows to offered services demands and existing networks physical topology. It often requires usage of creativity and working out new, non-standard solutions. Solutions, which could help in traffic flow supervision and in network load control dynamically.

Drawing common attention to atypical solutions for multi-criteria optimization, like ant algorithms, it is necessary and even essential to find new solutions and keep the market going, to find new ideas of resolving network problems. Such non-standard solutions in connection with new technologies, statistical researches and experience of engineers are able to give bright perspectives for development of telematic networks.

\section{REFERENCES}

Aiex, R. M., Resende, M. G. C., Pardalos, P. M. and Toraldo, G., 2005. Grasp with path relinking for the three-index assignment problem. INFORMS Journal on Computing, $17(2), 224-247$.

Balas, E. and Saltzman, M. J., 1991. An algorithm for the three-index assignment problem. Operations Research, 39(1), 150-161.

Burkard, R. E., Rudolf, R. and Woeginger, G. J., 1996. Three dimensional axial assignment problems with decomposable cost coefficients. Discrete Applied Mathematics, 65(1-3), $123-139$.

Crama, Y. and Spieksma, F. C. R., 1992. Approximation algorithms for three-dimensional assignment problems with triangle inequalities. European Journal of Operational Research, 60(3), 273-279.

Escamilla-Ambrosio, P. J. and Lieven, N., 2004. A multiple-sensor multiple-target tracking approach for the autotaxi system. IEEE Intelligent Vehicles Symposium, Parma, June 14-17, pp. 601-606.

Filipowicz, B., 1998. Mathematical modelling of decision issues - part I [in Polish]. AGH University of Science and Technology Press, Krakow.

Górecki, H., 2006. Optimization and control of dynamic systems [in Polish]. AGH University of Science and Technology Press, Krakow.

Huang, G. and Lim, A., 2006. A hybrid genetic algorithm for the three-index assignment problem. European Journal of Operational Research, 172(1), 249-257.

Karp, R.M., 1972. Reducibility among combinatorial problems. [in:] Miller, R. E., Thatcher, J. W. (Eds): Complexity of Computer Computations, Plenum Press, pp. 85-103.

Kuhn, H.W., 1955. The hungarian method for the assignment problem. Naval Research Logistics Quarterly, 2, pp. 83-97.

Merkle, D. and Middendorf, M., 2005. Swarm intelligence. [in:] Burke, E., Kendall, G. (Eds), Introductory Tutorials in Optimization, Search and Decision Support Techniques Methodology, Springer, pp. 401-435.

Owsinski, J. W. and Straszak, A., 2002. Information Society, operational research and management [in Polish]. EXIT Publishing House, Warszawa.

Zajdel, M. and Filipowicz, B., 2008. Multidimensional assignment problem as a tool for decision processes' support in company [in Polish]. Models of computer science engineering. Selected applications [in Polish], Volume 3, pp. 288-293. 